\title{
A History of the Arf-Kervaire Invariant Problem
}

\author{
Victor P. Snaith
}

"I know what you're thinking about," said Tweedledum; "but it isn't so, nohow." "Contrariwise," continued Tweedledee, "if it was so, it might be; and if it were so, it would be; but as it isn't; it ain't. That's logic."

-Through the Looking Glass, by Lewis Carroll (aka Charles Lutwidge Dodgson) [8]

Typing "Invariant theory" into Wikipedia yields the theory of functions like $x_{1} x_{2}+x_{1} x_{3}+x_{2} x_{3}$ which are unaltered by permuting the variables. In algebraic topology, particularly post-1950, a different notion of "invariant" emerged. This use of invariant (e.g., Hopf invariant, Arf-Kervaire invariant, $\lambda$-invariant) denotes an algebraic quantity that gives a partial answer to a topological question.

Often invariants in this sense are very technical, both in their context and in their construction. However, a very simple invariant occurs in the game of Nim ([31], pp. 36-38). In the 1960s this game was popular among students due to its enigmatic appearance in Alain Resnais's 1961 avant-garde movie L'Année Dernière à Marienbad.

A set of matchsticks is divided arbitrarily into several heaps. Two players play alternately. A play consists of selecting a heap and removing from it any (nonzero) number of matchsticks. The winner is the player whose move leaves no remaining matchsticks. The question which the Nim invariant answers is, If my opponent and I play out of our skins, will I win?

Suppose there are $k$ heaps of matchsticks of sizes $n_{1}, \ldots, n_{k}$. Recall that every positive integer $n$ can be written in one and only one way as the sum of distinct powers of 2 . This is called the binary

Victor P. Snaith is emeritus professor of mathematics at the University of Sheffield. His email address is v. snai th@ sheffield.ac.uk.

DOI: http://dx.doi.org/10.1090/noti1030 or dyadic formula for $n$. For example, $17=1+2^{4}$, $35=1+2+2^{5}$, and $60=2^{2}+2^{3}+2^{4}+2^{5}$. The binary formula may be depicted as a string of 0 's and 1 's that records in the $i$-th place whether or not $2^{i}$ appears in the binary formula for $n$. Here is a sample table of binary strings:

\begin{tabular}{|l|l|}
\hline \hline 17 & 10001 \\
\hline 35 & 110001 \\
\hline 60 & 001111 \\
\hline
\end{tabular}

If we express each of $n_{1}, \ldots, n_{k}$ as a row in a similar table of binary strings, we define the Nim invariant to be the string of integers given by the column sums. The Nim invariant of $17,35,60$ is $(2,1,1,1,2,2)$. If every entry in the Nim invariant is even (which we shall call the Nim condition) after a player's turn, then the answer to the question is yes; otherwise it is no.

If the Nim condition holds after Player A's turn, then either A has won or any move by Player B destroys the Nim condition. In particular, Player B has not won! Conversely, if the Nim condition does not hold after Player B's turn, then Player A can restore it by the following algorithm: Player A inspects the string of column sums from right to left to find the first odd column sum. Suppose this is the column corresponding to $2^{i}$. Then Player A chooses a row in which there is a 1 in the $i$-th column. Player A takes some matchsticks from the pile corresponding to this row. There is always a number of matchsticks which Player A can remove from this pile in order to restore the Nim condition. For example, in the table above, the $2^{3}$-column has an odd sum, and Player A finds a 1 in the bottom row. Subtracting $2^{3}+2=10$ from 60 changes the table to:

\begin{tabular}{|l|l|}
\hline \hline 17 & 10001 \\
\hline 35 & 110001 \\
\hline 50 & 010011 \\
\hline
\end{tabular}


whose column sum is $(2,2,0,0,2,2)$. With this algorithm Player A has restored the Nim condition and reduced the total number of matchsticks. Since Player B cannot win, playing perfectly, Player A must do so.

The Arf invariant, which occurred first in algebra [5], requires a little more mathematical background. Let $V$ be the $n$-dimensional vector space over the field $\mathbb{F}_{2}$ of two elements. In more concrete terms, let $V$ be the set of $n$-tuples $\underline{x}=\left(x_{1}, x_{2}, \ldots, x_{n}\right)$ in which each $x_{i}$ is equal to 0 or 1 . Recall that "addition" on $V$ is defined by setting $\underline{x}+y$ equal to the $n$-tuple whose $j$-th entry equals 0 if $x_{j}+y_{j}$ is even and equals 1 otherwise. $\mathbb{F}_{2}$ is the example in which $V$ consists of 1-tuples.

A quadratic form is a function $q: V \longrightarrow \mathbb{F}_{2}$ such that $q((0, \ldots, 0))=0$ and the associated function $Q: V \times V \rightarrow \mathbb{F}_{2}$ given by $Q(\underline{x}, \underline{y})=q(\underline{x}+\underline{y})-q(\underline{x})-$ $q(\underline{y})$ satisfies $Q(\underline{x}, \underline{y}+\underline{z})=Q(\underline{x}, \underline{y})+Q(\underline{x}, \underline{z})$. This function of two $V$-variables is called an $\mathbb{F}_{2}$-bilinear form. It is symmetric and symplectic, which means $Q(\underline{x}, y)=Q(y, \underline{x})$ and $Q(\underline{x}, \underline{x})=0$ for all $\underline{x}, y$ in $V$. The function $q$ is called a quadratic refinement of $Q$. Three examples when $n=2$ are given by $q\left(x_{1}, x_{2}\right)=x_{1} x_{2}, q^{\prime}\left(x_{1}, x_{2}\right)=x_{1}^{2}+x_{1} x_{2}+x_{2}^{2}$, and $q^{\prime \prime}\left(x_{1}, x_{2}\right)=\left(x_{1}+x_{2}\right) x_{2}$. For larger, even values of $n$, further quadratic forms may be made by applying one of $q, q^{\prime}$, or $q^{\prime \prime}$ to $\left(x_{2 i-1}, x_{2 i}\right)$ for $i=1,2, \ldots, n / 2$ and adding the results in $\mathbb{F}_{2}$.

Two quadratic forms $q_{1}$ and $q_{2}$ on $V$ are called equivalent if there is a bijective, linear change of coordinates which transforms $q_{1}$ into $q_{2}$. For example, when $n=2, q^{\prime \prime}$ is equivalent to $q$ via the coordinate transformation $\left(x_{1}, x_{2}\right) \mapsto\left(x_{1}+x_{2}, x_{2}\right)$.

A nonsingular quadratic form $q_{1}$ is one for which $n$ is even and is equivalent either to the sum of $n / 2$ copies of $q$ or to $q^{\prime}$ plus the sum of $(n-2) / 2$ copies of $q$. Hence, for each even integer $n$, there are just two equivalence classes of $q_{1}$ 's. The Arf invariant $c\left(q_{1}\right)$ lies in $\mathbb{F}_{2}$ and answers the question: To which equivalence class does the nonsingular quadratic form $q_{1}$ belong?

The definition of $c\left(q_{1}\right)$ given in [5] involves a complicated algebraic formula, which at first sight is not even well defined. In [7] Bill Browder used an amusing equivalent definition of the Arf invariant as the following "democratic invariant". The elements of $V$ "vote" for either 0 or 1 by the function $q_{1}$. The winner of the election (which is never a tie) is $c\left(q_{1}\right)$. Here is a table illustrating this for the three possibilities $q, q^{\prime}, q^{\prime \prime}$ when $V=\{(0,0),(0,1),(1,0),(1,1)\}$. Having equal Arf invariants, $q^{\prime \prime}$ and $q$ are equivalent, as we observed earlier, the vote being three to one in each case.

\begin{tabular}{|c|c|c|c|c|c|}
\hline \hline$x$ & $(0,0)$ & $(0,1)$ & $(1,0)$ & $(1,1)$ & value of $c$ \\
\hline \hline values of $q$ & 0 & 0 & 0 & 1 & 0 \\
\hline values of $q^{\prime}$ & 0 & 1 & 1 & 1 & 1 \\
\hline values of $q^{\prime \prime}$ & 0 & 1 & 0 & 0 & 0 \\
\hline
\end{tabular}

The Nim and Arf invariants fortuitously give a necessary and sufficient answer to their mathematical questions. As the mathematical subtlety of the question deepens, one must often settle for invariants which give only partial information. Algebraic topology is littered with examples of the calculational device known as a spectral sequence. A spectral sequence is an invariant (some poetic license may be needed here) whose output can often be highly ambiguous. Spectral sequences, like Marmite, are either loved or hated, and in the 1960s, graduate courses about them were regularly inflicted on unwilling students in a variety of research areas. One of the most famous is the Adams spectral sequence, invented in the 1950s by Frank Adams [1] (unfortunately universally abbreviated to ASS), which turns cohomological algebra into calculational information about stable homotopy groups. Typically a spectral sequence comprises an infinite family of abelian groups, calculable by an algebraic algorithm, together with maps between them called differentials. The ambiguity arises from the fact that. even if one knew the identity of all the differentials, the algorithm for their use yields information concerning only a filtration of the abelian groups which the spectral sequence is said "to compute". In algebraic topology, information squeezed from invariants may be hard won. One's motto should be "Do not expect too much of an invariant." We shall return to the ASS later.

Algebraic topology is generally believed to have begun with Poincaré in [26], which initiated the study of differentiable manifolds. Poincaré posed problems (e.g., the Poincaré Conjecture [24]) of generalizing to higher dimensions the success of the nineteenth-century geometers in classifying surfaces. His interest in manifolds stemmed partly from his study of the global properties of solution curves to differential equations on orientable surfaces and partly from his use of the method of Riemann surfaces in connection with complex function theory.

A differentiable manifold is a set of points in which each point lies in a coordinate patch modelled on the Euclidean space $\mathbb{R}^{n}$ consisting of $n$-tuples of real numbers, which is familiar from several-variable calculus courses. Where two coordinate patches overlap, the change-of-coordinates function is required to be highly differentiable in terms of the two sets of local coordinates. A differentiable map between two manifolds is a function $f: M \rightarrow N$ that is differentiable in terms of the local coordinates, and $f$ is a diffeomorphism if it is one-one and onto and has a differentiable inverse map.

Poincaré gave sample methods for the construction of manifolds. Among the methods for studying 
manifolds up to diffeomorphism which he introduced are homology, the fundamental group, and the relation of cobordism. His successes include Poincaré duality for compact, closed, and oriented manifolds.

To appreciate the need for invariants in algebraic topology, consider the $n$-dimensional sphere $S^{n}$ consisting of the points $\underline{x}=\left(x_{0}, x_{1}, \ldots, x_{n}\right)$ in $R^{n+1}$ such that $x_{0}^{2}+x_{1}^{2}+\cdots+x_{n}^{2}=1$. Hence $S^{n}$ with two coordinate patches consisting of slightly enlarged upper and lower hemispheres is the simplest differentiable manifold after Euclidean space. In professional jargon, it is a smooth compact manifold without boundary. The simplest problem one might pose is the classification of continuous maps $f: S^{n} \rightarrow S^{m}$. Clearly we have the identity map when $n=m$, and in all cases we have the constant maps. In order to render the problem a little more tractable, we introduce the notion of based homotopy classes of maps. Suppose that each manifold $M$ that we shall consider comes equipped with a base point within it, usually denoted by $* \in M$. Henceforth we shall consider only based continuous maps between manifolds, meaning that base point is mapped to base point. Two continuous, based maps $f_{0}, f_{1}: M \longrightarrow N$ are homotopic if there is a continuous map $H:[0,1] \times M \rightarrow N$ such that $H(0, \underline{x})=f_{0}(\underline{x}), H(1, \underline{x})=f_{1}(\underline{x})$, and for all $t$ we have $H(t, *)=*$. In order to ensure that invariants involving based homotopy classes of maps do not depend on the choice of $*$, we shall assume from now on that each of our manifolds $M$ is path connected so that there is a continuous path between any two choices of $*$. Of course, $S^{n}$ is path connected if $n \geq 1$.

Ever optimistic, we would like an invariant that gives an answer to the question: Is a continuous, based map $f: S^{n} \rightarrow S^{m}$ not homotopic to a constant map? When $m=n$, Poincaré already had the answer: the degree of $f$ must be nonzero. More precisely, in this case the homology group $H_{n}\left(S^{n}\right)$ is equal to $\mathbb{Z}$, the integers, and $f$ induces a homomorphism $f_{*}$ from the group $H_{n}\left(S^{n}\right)$ to itself, which must be multiplication by an integer, the degree of $f$. When $n<m$, approximation theorems allow us to replace $f$ by a based map $h$ which is homotopic to $f$ while also being highly differentiable. The differential techniques of Poincaré show that the image of $h$ misses a point, which easily implies that $h$ (and therefore $f$ ) is homotopic to a constant map. The case when $m<n$ is considerably harder and remains unsolved in general for want of sufficiently powerful invariants.

Prior to the 1950s, although there had been some wonderful post-Poincaré discoveries during the intervening years (e.g., fixed point theorems), it seems to me that algebraic topology was in a state analogous to that of calculus in 225 B.C. Recall that, in a series of essays ([4]; for example, see [25]), Archimedes had successfully used ingenious ad hoc arguments, which prefigure integral and differential calculus, in order to calculate ratios of curve lengths, areas and volumes, and to find centers of gravity of selected geometrical shapes. His methods included dissecting the shapes in the style of integral calculus, then calculating the ratio under consideration up to an error which was assumed infinitesimally small. However, the method did not amount to a theory, since Archimedes could make it work only for a small set of judiciously chosen shapes. Similarly, for example, in algebraic topology prior to [13], the search for a continuous dimension-lowering map between spheres which is not homotopic to the constant map had drawn a blank.

In retrospect, finding an example was rather easy. Consider a point $P$ in $S^{3}$ given by a pair $\left(z_{1}, z_{2}\right)$ of complex numbers whose squared absolute values add up to 1 . Then the point $\left(e^{\theta \sqrt{-1}} Z_{1}, e^{\theta \sqrt{-1}} Z_{2}\right)$ draws a circle on the 3 -sphere passing through $P$. The set which has one point for each of these circles is $\mathbb{C P}^{1}$, the space of complex lines through the origin in the space of pairs of complex numbers, which is homeomorphic to the 2-sphere. Sending $P$ to the circle through it gives a differentiable map between manifolds $h: S^{3} \longrightarrow S^{2}$. On the other hand, the problem of showing that this map is not homotopic to the constant map is far from easy. In fact, Heinz Hopf originally established this by ad hoc ingenuity based on the observation that the inverse images of any two distinct points on $S^{2}$ are linked circles.

What was needed in order to systematize this sort of result was a calculable invariant-in the above case, the Hopf invariant. The Hopf invariant is an integer that is associated to any continuous, based map of the form $f: S^{2 n-1} \longrightarrow S^{n}$ for $n \geq 1$. Later, with the construction of cohomology operations by Norman Steenrod, it became possible to define the parity of the Hopf invariant for any $g: S^{m} \longrightarrow S^{n}$ (with $n \gg 0$ ) and to show that the only possibilities for odd Hopf invariants occur when $m-n+1$ is a power of two ([35], p. 12). The full story is the famous result, due originally to Frank Adams ([2]; see also [3], [32]), which shows that only the cases $m-n=1,3,7$ admit homotopy classes with odd Hopf invariant. As we shall see later, the Arf-Kervaire problem is the next natural obstacle after [2].

The mid-1950s was an exciting time for algebraic topology, ushering in a golden age of the construction of invariants and the development of methods for their calculation. Sociologically, one can see what was to come by looking for the 
presence of mathematical luminaries in the photograph of the 1956 International Symposium on Algebraic Topology held in Mexico City in honor of Solomon Lefschetz. Alternatively, one may reflect that sixty years after [26] there was still precious little known about the differential structure of manifolds-even the sphere. Then, in [21], enters the first of the exotic spheres. An exotic sphere is a smooth manifold that is homeomorphic to, but not diffeomorphic to, a sphere. At that time such manifolds were very hard to find. In fact, in dimension three, Munkres, Smale, and Whitehead independently had shown that there were no nontrivial exotic spheres. It is worth going into some detail at this point to get the flavor of just how adventurous the construction of invariants was destined to become.

To get any further we shall need to collect mathematical objects in groups, and usually we prefer abelian groups. For example, every manifold has abelian homology groups $H_{S}(M)$ and cohomology groups $H^{s}(M)$ of various flavors, as well as homotopy groups $\pi_{r}(M)$ for $r \geq 1$. The latter is the set of homotopy classes of continuous, based maps $f: S^{r} \rightarrow M$. Addition of $g_{1}$ and $g_{2}$, which is abelian if $r \geq 2$, is accomplished by pinching $S^{r}$ to a point at the equator to form two copies of $S^{r}$ (joined at one common point, the base point) and using $g_{1}$ and $g_{2}$ to map the two $S^{r}$ 's to $M$.

A compact smooth $n$-dimensional manifold without boundary (i.e., closed and, by our convention, path connected) $M^{n}$ is orientable if its top-dimensional integral homology group $H_{n}\left(M^{n}\right)$ is isomorphic to the integers and is oriented if a generator has been chosen.

There are some closed orientable $M^{7} \mathrm{~s}$ which are easy to describe, whose construction was discovered by John Milnor [21]. One starts with a continuous, based map $f: S^{3} \rightarrow S O(4)$. Here $S O(4)$ is the group of $4 \times 4$ orthogonal matrices of determinant one with the identity matrix as base point. If $\underline{x}=\left(x_{1}, x_{2}, x_{3}, x_{4}\right)$ is a point of $\mathbb{R}^{4}$ which is in $S^{3}$ and $Y$ is a $4 \times 4$ matrix in $S O(4)$, then the matrix product $\underline{x} Y$ is again a point on $S^{3}$. The 4-dimensional disk is the set of points in $\mathbb{R}^{4}$ such that $x_{1}^{2}+x_{2}^{2}+x_{3}^{2}+x_{4}^{2} \leq 1$ with boundary equal to $S^{3}$. Take two copies of the product $D^{4} \times S^{3}$ and glue them together along the boundary $S^{3} \times S^{3}$ according to the recipe $(\underline{x}, y) \mapsto(y, \underline{x} f(y))$. This process is called "plumbing". When $f$ sends each point to the identity we see that plumbing results in the space $S^{7}$. In addition, $f$ may be replaced by a homotopic map that is highly differentiable, in which case Milnor showed that the plumbing space is a 7-dimensional differentiable manifold $M^{7}$. Potentially there are lots of homotopy classes of $f^{\prime}$ 's for use in the above construction, since it is known that the homotopy group $\pi_{3}(S O(4))$ is equal to $\mathbb{Z} \oplus \mathbb{Z}$. Finally, in order to show that some of these $M^{7}$ 's are exotic spheres, Milnor had to construct an entirely new type of invariant, the $\lambda$-invariant. By the calculations of [36], there exists an 8-manifold $B^{8}$ whose boundary is $M^{7}, \partial B^{8}=M^{7}$. An ingenious argument involving $B^{8}$ was used in [21] to construct the $\lambda$-invariant which identified some of these $M^{7}$ s as exotic 7 -spheres.

The construction of [21] was streamlined and systematized in [23] using the homotopy groups of larger special orthogonal groups to construct $M^{4 k-1} \mathrm{~S}$ which were the boundary of a $B^{4 k}$. The original strategy is generalized by applying the full force of Hirzebruch's signature theorem, which appeared in 1956 ([11]; see also [12]), to the manifold $B^{4 k}$ to extend the $\lambda$-invariant and thereby count the number of distinct exotic spheres. For example, the method showed that $S^{31}$ has more that $16 \times 10^{6}$ differentiable structures!

In [17], diffeomorphism classes of $n$-dimensional exotic spheres were used to define a finite abelian group denoted by $\Theta_{n}$. If $M_{1}$ and $M_{2}$ are smooth oriented manifolds, each homeomorphic to $S^{n}$, then their connected sum $M_{1} \# M_{2}$ is the space formed by removing a small $n$-dimensional disk from each and pasting together the resulting boundaries. Clearly $M_{1} \# M_{2}$ is homeomorphic to $S^{n}$. In [17] it is shown that it can be given the structure of a smooth oriented manifold. Connected sum gives the "addition" operation in the group $\Theta_{n}$ such that $M_{1} \# S^{n}$ is diffeomorphic to $M_{1}$. In $\Theta_{n}$ the negative of the class of $M_{1}$ is represented by $\left(-M_{1}\right)$, the manifold $M_{1}$ but with the opposite orientation.

The improved $\lambda$-invariant gives a homomorphism that takes its values in the abelian quotient group $\mathbb{Q} / \mathbb{Z}$ whose elements are the rational numbers $q$ in the range $0 \leq q<1$. This group has an infinite number of finite abelian groups within it, making it an ideal target for estimating the size of $\Theta_{n}$. For example, from [17], for low values of $k$ we have the following table:

\begin{tabular}{|c|c|c|c|}
\hline \hline$k$ & 7 & 11 & 15 \\
\hline$\left|\Theta_{4 k-1}\right|$ & 28 & 992 & 16256 \\
\hline
\end{tabular}

Before leaving exotic spheres (to which we shall return later) it should be pointed out that the methods of [17] successfully computed the order of $\Theta_{n}$ for many dimensions, not just those of the form $n=4 k-1$, and their results essentially metamorphosed the problem into questions about the stable homotopy groups of spheres. As it happened, fortunately, the 1950s and 1960s saw considerable advances in the calculational techniques of homotopy theory.

Next we turn to stable homotopy groups of spheres. For $t \geq 2$ there is a homomorphism of abelian groups $\Sigma: \pi_{t}\left(S^{n}\right) \longrightarrow \pi_{t+1}\left(S^{n+1}\right)$ called the 
suspension homomorphism. One considers each latitudinal level in $S^{r+1}$ (resp. $S^{n+1}$ ) as a copy of $S^{r}\left(\right.$ resp. $\left.S^{n}\right)$ and defines $\Sigma(f)$ to be the homotopy class of the map which maps levelwise via $f$. The map $\Sigma$ is a bijection provided that $t \leq 2 n-2$ and the stable homotopy group $\pi_{r}^{S}\left(S^{0}\right)$ is defined to be $\pi_{r+m}\left(S^{m}\right)$ for $m \gg 0$. Notice that $\pi_{r}^{S}\left(S^{0}\right)$ makes sense even when the integer $r$ is negative and, in fact, this group is zero for $r<0$, finite for $r>0$.

There are two famous constructions which land in the abelian group $\pi_{r}^{S}\left(S^{0}\right)$. The $J$-homomorphism $J: \pi_{r}(S O) \longrightarrow \pi_{r}^{S}\left(S^{0}\right)$ is simple to describe. Recall that multiplication by a matrix $Y$ in the special orthogonal group $S O(n)$ maps the sphere $S^{n-1}$ to itself. A continuous map $h: S^{r} \rightarrow S O(n)$ induces a map $\tilde{h}$ from $S^{r} \times S^{n-1}$ to $S^{n-1}$ via the formula $\tilde{h}(\underline{x}, y)=y h(\underline{x})$. The following construction due to Heinz Hopf applied to $\tilde{h}$ produces a continuous map $J(h): S^{r+n} \longrightarrow S^{n}$. Let $(\underline{x}, \underline{y})$ be a point in $\mathbb{R}^{r+n+1}$ with $\underline{x}=\left(x_{1}, \ldots, x_{r+1}\right) \in \mathbb{R}^{r+1}$ and $\underline{y}=$ $\left(y_{1}, \ldots, y_{n}\right) \in \mathbb{R}^{n}$. The standard norm of $|\underline{x}|$ is the positive square root of $x_{1}^{2}+\cdots+x_{r+1}^{2}$ and similarly for $|\underline{y}|$. If $(\underline{x}, \underline{y}) \in S^{r+n}$, then $|\underline{x}|^{2}+|\underline{y}|^{2}=1$ and $(|\underline{x}|, \bar{h}(\underline{x} /|\underline{x}|, \underline{y} /|\underline{y}|)$ is (almost) a point of the cylinder $[0,1] \times S^{n-1}$. The problems occur when $|\underline{x}|=0,1$. In these cases the formula gives $(0, h(?, \underline{y}))$ and $(1, h(\underline{x}, ? ?))$ where ? and ?? indicate an unspecified point of $S^{r}$ and $S^{n-1}$ respectively. However, if we compose with the map from the cylinder to $S^{n}$ which sends $(t) \times S^{n-1}$ by the identity map to the $S^{n-1}$ at latitudinal level $t$ when $0<t<1$ and sends every point with level $t=0$ or $t=1$ to the south or north poles respectively, we obtain a well-defined continuous map $J(h)$.

The homotopy groups of $S O(n)$ for large $n$ were famously calculated by Raoul Bott [6]. By work (in the 1960s and 1970s) of J. F. Adams, M. F. Atiyah, M. Mahowald, D. Sullivan, D. G. Quillen, and others, the image of $J$ is known.

The second famous construction, due to Lev Pontrjagin [27], [28] and René Thom [36], describes $\pi_{r}^{S}\left(S^{0}\right)$ in terms of (framed) manifolds. Interestingly, Pontrjagin introduced this construction in order to compute stable homotopy groups via the geometry of manifolds, while Thom generalized the construction in order to reduce the analysis of the cobordism relation between manifolds to the calculation of stable homotopy groups.

We shall require only the special case in which the manifold $\Sigma^{k}$ is a $k$-dimensional exotic sphere. Consider the circle $S^{1}$ embedded as a subset of $\mathbb{R}^{2}$ in the usual manner as the subset of points whose norm is equal to one. The normal to the circle at $\underline{x}=\left(x_{1}, x_{2}\right)$ comprises all the scalar multiples $\lambda \underline{x}$ where $\lambda$ is any real number. The normal unit disk bundle comprises the union, as $\underline{x} \in S^{1}$ varies, of the points in the normal at $\underline{x}$ within (a chosen small) unit distance of $\underline{x}$. In this example the unit disk bundle may be realized by the subset of $\mathbb{R}^{2}$ consisting of points $\lambda \underline{x}$ where $\underline{x} \in S^{1}$ and $1 / 2 \leq \lambda \leq 1$. Hence $\lambda \underline{x} \mapsto(\underline{x}, \lambda)$ gives a homeomorphism of the normal unit disk bundle with $S^{1} \times[1 / 2,1]$. Identify $S^{1}$ with the space obtained from $[1 / 2,1]$ by gluing its endpoints together. Sending $(x, \lambda)$ to $\lambda$ induces a continuous map from the disk bundle to $S^{1}$ that sends each point on the boundary of the disk bundle to the base point. Considering $S^{2}$ as the union of $\mathbb{R}^{2}$ with a point at infinity (as in the classical stereographic projection used by cartographers), the map extends continuously to all of $S^{2}$ by sending " $\infty$ " and all points of $\mathbb{R}^{2}$ outside or on the boundary of the disk bundle to the base point.

More generally, if we embed an exotic sphere $\Sigma^{k}$ as a differentiable submanifold of $\mathbb{R}^{k+m}$ for $m \gg 0$, there is a homeomorphism, called a framing, of the normal disk bundle with $S^{k} \times D^{m}$. In this case the Pontrjagin-Thom construction yields a continuous map $S^{k+m} \rightarrow S^{m}$. Remarkably, the PontrjaginThom construction gives a well-defined element of $\pi_{k}^{S}\left(S^{0}\right)$ depending only on the cobordism class of the framed manifold $\Sigma^{k}$. I shall skip the definition of cobordism of manifolds and other manifoldrelated structures-suffice it to repeat that the notion was introduced by Poincaré and to add that the efficacy of the Pontrjagin-Thom construction is intimately related to Poincaré's observation that the inverse image of a regular point is a manifold.

However, the manifold $\Sigma^{k}$ does not know about embeddings and framings, so we do not receive a homomorphism from $\Theta_{k}$ to $\pi_{k}^{S}\left(S^{0}\right)$. On the other hand, it can be shown that varying the framing changes the element of $\pi_{k}^{S}\left(S^{0}\right)$ only by the addition of an element $J(z)$ in the image of the $J$-homomorphism. Therefore we obtain a homomorphism $\tau_{k}: \Theta_{k} \longrightarrow \pi_{k}^{S}\left(S^{0}\right) / \operatorname{Im}(J)$ where the elements of the latter abelian group are the subsets of $\pi_{k}^{S}\left(S^{0}\right)$ of the form $\{x+J(z)\}$ as $z$ varies through $\pi_{k}(S O(t))$ for $t \gg 0$ and $x$ is an element of $\pi_{k}^{S}\left(S^{0}\right)$. In algebraic topology this is a very important homomorphism about which almost everything is now known. Some of the final missing pieces of the jigsaw are provided by the Arf invariant in a topologically embellished form known as the Arf-Kervaire invariant.

Using the Arf invariant, Kervaire [16] defined an $\mathbb{F}_{2}$-valued invariant for certain compact, topological manifolds and applied it to exhibit a 10-dimensional topological manifold that does not admit any differentiable structure!

The following construction from [7] extends this definition to any framed, closed $(4 l-2)$ manifold. It hinges on the ingenious construction of a quadratic form that takes values in a stable homotopy group. For any space $X$ with a base point, 
the stable homotopy groups $\pi_{k}^{S}(X)$ are defined in an analogous manner to the case $X=S^{0}$, which we met earlier.

The Eilenberg-MacLane space $K(\mathbb{Z} / 2, k)$ is characterized up to homotopy equivalence by having only one nontrivial homotopy group, namely, $\pi_{k}(K(\mathbb{Z} / 2, k))$, which has precisely two elements. The mod 2 singular cohomology group of $M$, denoted by $H^{k}(M ; \mathbb{Z} / 2)$, is the group of homotopy classes of continuous, based maps from $M$ to $K(\mathbb{Z} / 2, k)$.

Suppose we are given a framed manifold $M^{2 k}$ and a cohomology class $a \in H^{k}(M ; \mathbb{Z} / 2)$. In this case the Pontrjagin-Thom map takes the form $S^{2 k+N} \rightarrow \frac{S^{N} \times M}{* \times M}$, whose target is the product $S^{N} \times M$ with any point whose $S^{N}$-coordinate is equal to the north pole identified with the base point. Composing this with any map whose homotopy class represents $a$ yields a stable homotopy class $q_{M, t}(a) \in \pi_{2 k}^{S}(K(\mathbb{Z} / 2, k))$. This stable homotopy group is isomorphic to $\mathbb{F}_{2}$.

This gives a nonsingular quadratic form $q_{M, t}$ on $V=H^{k}(M ; \mathbb{Z} / 2)$, depending on the framing $t$, and the Arf-Kervaire invariant of $(M, t)$ is $c\left(q_{M, t}\right) \in \mathbb{F}_{2}$. A celebrated result of Browder [7] asserts that the Arf-Kervaire invariant of a framed manifold $M^{4 l-2}$ is trivial unless $l=2^{s}$ for some $s$. Via the Pontrjagin-Thom construction, the Arf-Kervaire invariant may be considered as a homomorphism $\operatorname{Arf}_{n}: \pi_{2^{n}-2}^{S}\left(S^{0}\right) \longrightarrow \mathbb{Z} / 2$ for $n \geq 2$. Since 1969 one of the major questions in stable homotopy theory has been: $\operatorname{Is~}_{\operatorname{Arf}} n$ nonzero?

The Arf-Kervaire invariant has a very important influence on the behavior of $\tau_{k}$ in the following manner:

Theorem. If $\operatorname{Arf}_{4 l+2}=0$, then $\tau_{4 l+2}$ is surjective and the kernel of $\tau_{4 l+1}$ consists of two elements. If $\operatorname{Arf}_{4 l+2} \neq 0$, then the cokernel of $\tau_{4 l+2}$ is a group with precisely two elements.

In view of the Pontrjagin-Thom result, the last part of the above theorem may be rephrased geometrically as the fact that, in dimension $4 l+2$, the Arf-Kervaire invariant is the only obstruction to finding an exotic sphere in a framed cobordism class. This is the sort of manifold-theoretic statement that would have appealed to Poincaré; in particular, he would have surely been delighted to see the problem resolved. In 2009, Mike Hill, Mike Hopkins, and Doug Ravenel (or HHR for short) posted a proof of a result which almost completely did just that.

Theorem ([10]; see also [9] and [20]). The homomorphism $\operatorname{Arf}_{n}$ is zero for $n \geq 8$.

This result leaves open only dimension 126, and my personal opinion would be that there is no element in $\pi_{126}^{S}\left(S^{0}\right)$ having odd Arf-Kervaire invariant. In fact, settling the problem in this dimension might be within the scope of a computerassisted computation such as that of [18]; see also [19].

When the dust settled following the appearance of [10], the outcome was "mostly, framed manifolds of odd Arf-Kervaire invariant do not exist," as predicted by Tweedledum and Tweedledee in the opening quotation!

To get even the vaguest feel for what goes into the HHR result (the argument is analogous to Doug Ravenel's odd primary result [29]) it is necessary to return to the promised account of Adams spectral sequences and their intrinsic ambiguities. The classical ASS [1] begins with the discovery of the Steenrod algebra $\mathcal{A}$ of natural operations on the $\mathbb{F}_{2}$-cohomology of spaces. This is an infinite-dimensional abelian group which comes as a direct sum of finite-dimensional pieces, each indexed by a cohomological dimension. Thus far, $\mathcal{A}$ is an example of a graded abelian group. Composing two operations gives another one, and this "product" turns $\mathcal{A}$ into a graded ring. In fact, Milnor showed that $\mathcal{A}$ has the additional structure of a graded Hopf algebra [22]. One may perform elaborate, algorithmic homological algebra calculations with a graded Hopf algebra, and the classical ASS begins with the bigraded (like a graded one, but the pieces come indexed by pairs of integers) Ext-ring $\operatorname{Ext}_{\mathcal{A}}^{s, t}\left(\mathbb{F}_{2}, \mathbb{F}_{2}\right)$. The precise definition need not concern us, but here is a picture of $\operatorname{Ext}_{\mathcal{A}}^{s, t}\left(\mathbb{F}_{2}, \mathbb{F}_{2}\right)$ for small values of $s$ and $t$ ([30], Theorem 3.2.11).

For the range $t-s \leq 11$ and $s \leq 7$ the group $\operatorname{Ext}_{\mathcal{A}}^{s, t}(\mathbb{Z} / 2, \mathbb{Z} / 2)$ is a copy of $\mathbb{F}_{2}$ wherever an element is depicted in the following table and is zero elsewhere. The vertical coordinate is $s$ and the horizontal is $t-s$.

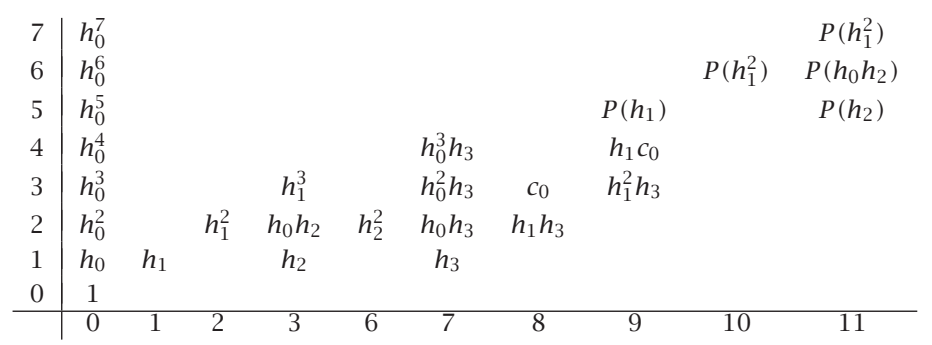

The differentials in the ASS are homomorphisms $d_{2}, d_{3}, \ldots$ from bigrading $(s, t)$ to $(s+r, t+r-1)$. Inductively the differentials are used to compute new tables of homology groups $E_{3}^{s, t}, E_{4}^{s, t}, \ldots$ Eventually the group in bigrading $(s, t)$ stabilizes and can then tell us something about the 2-Sylow subgroup of $\pi_{j}^{S}\left(S^{0}\right)$ for $t-s=j>0$ consisting of those elements whose order is a power of 2 . The simplest case occurs when the differentials are zero, which is the case in the range of dimensions in our table. 
For example, the occurrence of four $\mathbb{F}_{2}$ 's when $t-s=7$ tells us that the 2-Sylow subgroup of $\pi_{7}^{S}\left(S^{0}\right)$ has 16 elements, but it does not tell us that the subgroup is cyclic of order 16. On the other hand, when $t-s=1,2$ there is only one $\mathbb{F}_{2}$, and in fact $\pi_{1}^{S}\left(S^{0}\right)$ and $\pi_{2}^{S}\left(S^{0}\right)$ are both isomorphic to $\mathbb{F}_{2}$. For the record, the 2-Sylow subgroups of $\pi_{8}^{S}\left(S^{0}\right)$ and $\pi_{9}^{S}\left(S^{0}\right)$ are $\mathbb{F}_{2}$-vector spaces with 4 and 8 elements, respectively.

The appearances of $h_{1}, h_{2}, h_{3}$ and $h_{1}^{2}, h_{2}^{2}, \ldots$ in the table correspond respectively to the existence of maps of Hopf invariant one [2] and Arf-Kervaire invariant one [7].

I have included this excessive computational detail involving the classical Adams spectral sequence in low dimensions in order to impress on the reader just how badly the ambiguities ramify when one is trying to show, for example, that $h_{7}^{2}$ in dimension 254 (and bidegree $\left(2,2^{8}\right)$ ) survives despite the effect of all the differentials.

Since the 1960s the fans of ASS developed supercharged models [30] for which the input was a generalized cohomology theory. It is not possible here to go into the formidable technical details of the HHR theorem. Suffice it to say that the HHR result is based on the very ingenious discovery of a new generalized cohomology theory, which we shall denote by $L^{*}(-)$. It has the property that $L^{*}$ gives rise to a generalized Adams spectral sequence that comes with a map from the classical ASS and is sufficiently simple to compute so that one can show that a map $\theta: S^{r+2^{j}-2} \longrightarrow S^{r}$ with Arf-Kervaire invariant one induces a nonzero homomorphism $L^{r}\left(S^{r}\right) \longrightarrow$ $L^{r}\left(S^{r+2^{j}-2}\right) \cong L^{0}\left(S^{2^{j}-2}\right)$. This amounts to choosing $L^{*}$ so that the differentials in its generalized Adams spectral sequence are simple enough to control. Incredibly, HHR manage to accomplish all this and in addition find $L^{*}$ which satisfies $L^{r+2}\left(S^{r}\right)=0$ and $L^{m+256}\left(S^{n}\right) \cong L^{m}\left(S^{n}\right)$ for all $m, n$. This is curtains for the Arf-Kervaire invariant, because, for example, $L^{0}\left(S^{254}\right) \cong L^{256}\left(S^{254}\right)=0$ !

A result of Stewart Priddy and Dan Kahn enables one to give equivalent rephrasings of the Hopf invariant and the Arf-Kervaire invariant problems. Recall that real projective space $\mathbb{R}^{\mathbb{P}^{n}}$ is the topological space whose points consist of the antipodal pairs $\{\underline{x},-\underline{x}\}$, where $\underline{x}$ is a point on $S^{n}$. The Kahn-Priddy theorem [15] permits one to restate properties of $\pi_{k}^{S}\left(S^{0}\right)$ in terms of $\pi_{k}^{S}\left(\mathbb{R}^{n}\right)$ for $n \gg 0$. For example, the Kahn-Priddy reformulation implies Browder's original result that framed manifolds of Arf-Kervaire invariant one can occur only in dimensions of the form $2^{j}-2$ [34]. Collapsing $\mathbb{R}^{2 n}$ to the base point gives a continuous map $\pi: \mathbb{R} \mathbb{P}^{2 n+1} \rightarrow S^{2 n+1}$. The existence of maps of odd Hopf invariant, together with the Kahn-Priddy theorem, implies that, for any generalized cohomology or homology theory, $\pi$ behaves as if there is a map $f$ in the opposite direction such that the degree of $\pi \cdot f$ is odd. In [3] Ktheory, which is a generalized cohomology theory, is used to give a simpler proof of Adams's original theorem about the nonexistence of maps of odd Hopf invariant [2]. To each generalized cohomology theory there corresponds a generalized homology theory, which for K-theory is called connective K-homology. The connective K-homology proof in terms of the above Hopf invariant reformulation is so simple that I gave it in the midst of a book review [32]!

The Arf-Kervaire invariant problem is equivalent to posing the analogue of the above with $\mathbb{R} \mathbb{P}^{2 n+1}$ replaced by $\mathbb{R}^{2 n}$ ([33], [34]). In this sense, as I mentioned earlier, the Arf-Kervaire invariant is the next natural obstacle after the Hopf invariant.

Constructing elements of $\pi_{k}^{S}\left(S^{0}\right)$ in terms of continuous maps between spheres or in terms of framings on manifolds is a hard way to make a living.

As an alternative, I shall conclude by sketching a method that constructs a few elements of odd Arf-Kervaire invariant using manifolds that are known to be frameable but for which no specific framing need be given [33]. The infinitedimensional projective space $\mathbb{R} \mathbb{P}^{\infty}$ is just the union of all the $\mathbb{R}^{\mathbb{P}^{n}}$ s. It is also the classifying space $B O(1)$ of the group of $1 \times 1$ orthogonal matrices (aka the group with two elements). The group of $m \times m$ orthogonal matrices $O(m)$ has a classifying space $B O(m)$. A manifold $M^{2 t}$ together with a continuous map $E: M^{2 t} \longrightarrow B O(m)$ for $m \geq 2$ possesses a second Stiefel-Whitney class $w_{2}(E)$ in $H^{2}\left(M^{2 t} ; \mathbb{Z} / 2\right)$ and a fundamental class $[M]$ which is the only nonzero element of the top-dimensional homology group $H_{2 t}(M ; \mathbb{Z} / 2)$. Cohomology has a product that allows one to define an element $w_{2}(E)^{t}$ in $H^{2 t}(M ; \mathbb{Z} / 2)$. The pairing between top-dimensional cohomology and homology yields an element of $\mathbb{F}_{2}$, denoted by $\left\langle w_{2}(E)^{t},\left[M^{2 t}\right]\right\rangle$. In [33] it is shown that if $M^{2 t}$ is frameable and $\left\langle w_{2}(E)^{t},\left[M^{2 t}\right]\right\rangle$ is odd, then there exists an element of $\pi_{2 t}^{S}\left(S^{0}\right)$ with odd Arf-Kervaire invariant.

This result applies to each of the manifolds $M^{2 t}=\mathbb{R} \mathbb{P}^{t} \times \mathbb{R} \mathbb{P}^{t}$ for $t=1,3,7$ because $\mathbb{R} \mathbb{P}^{1}, \mathbb{R}^{3}$ and $\mathbb{R P}^{7}$ are Lie groups (hence frameable) and each $M^{2 t}$ is a subspace of $B O(2)$. In fact, there are nonstandard framings on these three Lie groups that give rise to the Hopf invariant one elements which are depicted by $h_{1}, h_{2}$, and $h_{3}$ in the classical ASS. Taking the Cartesian product of each of these with itself gives another construction of the Arf-Kervaire invariant one elements in dimensions 2,6 , and 14 , depicted by $h_{1}^{2}, h_{2}^{2}$, and $h_{3}^{2}$ in the classical ASS. 
Let $C$ denote the Riemann surface obtained by putting a thin tube around each edge of a cube. The natural action of $D_{8}$, the dihedral group of order 8 , on the cube induces a free $D_{8}$-action on $C$. Since $D_{8}$ is the 2-Sylow subgroup of the permutations on four objects, $D_{8}$ also acts on $\left(\mathbb{R P}^{7}\right)^{4}$. Let $D_{8}$ act simultaneously on the two factors of $C \times\left(\left(\mathbb{R} \mathbb{P}^{7}\right)^{4}\right)$. This is a free action with eight points in each orbit. Form the manifold $M^{30}=C \times_{D_{8}}\left(\left(\mathbb{R P}^{7}\right)^{4}\right)$, which has one point for each $D_{8}$-orbit. A simple calculation in K-theory [33] shows that $M^{30}$ is frameable (first noticed by John Jones [14]) and $M^{30}$ is a subspace of $B O(4)$.

Each of these examples gives an odd value for $\left\langle w_{2}(E)^{t},\left[M^{2 t}\right]\right\rangle$ and hence shows the existence of odd Arf-Kervaire invariants in dimensions 2, 6, 14, and 30 .

\section{References}

[1] J. F. ADAMS, On the structure and applications of the Steenrod algebra, Comm. Math. Helv. 32 (1958), 180-214.

[2] _ On the nonexistence of elements of Hopf invariant one, Annals of Math. 72 (1960), 20-104.

[3] J. F. AdAms and M. F. ATIYAH, K-theory and the Hopf invariant, Quart. J. Math. Oxford (2) 17 (1966), 31-38.

[4] Archimedes of Syracuse, Quadrature of the Parabola, Sphere and Cylinder, Spiral Lines, Conoids and Spheroids and The Method, privately circulated palimpsests (circa $225 \mathrm{BC}$ ).

[5] C. ARF, Untersuchungen über quadratische Formen in Körpern der Charakteristik 2 (Teil I), J Reine. Angew. Math. 183 (1941), 148-167.

[6] R. Bотт, The stable homotopy of the classical groups, Ann. Math. 70 (1959), 313-337.

[7] W. BROWDER, The Kervaire invariant of framed manifolds and its generalizations, Annals of Math. (2) 90 (1969), 157-186.

[8] L. CARroll, The Complete Works of Lewis Carroll (text Rev. Charles Lutwidge Dodgson, intro. Alexander Woollcott, illus. John Tenniel), The Nonesuch Press, London, 1940.

[9] M. A. Hill, M. J. Hopkins, and D. C. RAVENEL, Fixed point spectra of finite subgroups of $\mathbf{S}_{n}$, preprint available on webpage http://math. rochester. edu/u/faculty/doug/ preprints.html.

[10] , On the nonexistence of elements of Kervaire invariant one, arXiv:0908.3724v1[math.AT] (26 Aug 2009).

[11] F. HIRZEBRUCH, Neue topologische Methoden in der algebraischen Geometrie; Ergeb. Math. Band 9, Springer Verlag, 1962.

[12] _ Topological Methods in Algebraic Geometry, Grund. Math. Wiss. Band 131, Springer Verlag, 3rd ed.,1966.

[13] H. Hopf, Über die Abbildungen von Sphären auf Sphären niedriger Dimension, Fund. Math. 25 (1935), 427-440.

[14] J. D. S. JonES, The Kervaire invariant of extended power manifolds, Topology 17 (1978), 249-266.
[15] D. S. KAHN and S. B. PRIDDY, Applications of the transfer to stable homotopy theory, Bull. Amer. Math. Soc. 741 (1972), 981-987.

[16] M. A. KervaIRE, A manifold which does not admit any differentiable structure, Comm. Math. Helv. 34 (1960), 256-270.

[17] M. A. Kervaire and J. W. Milnor, Groups of homotopy spheres. I, Annals of Math. (3) 77 (1963), 504-537.

[18] S. O. Kochman, Stable Homotopy Groups of Spheres-A Computer Assisted Approach, Lecture Notes in Math., 1423, Springer-Verlag, 1990.

[19] S. O. Kochman and M. E. MaHOWALD, On the computation of stable stems, The Čech Centennial (Boston, MA, 1993), Contemp. Math., 181 Amer. Math. Soc., 1995, 299-316.

[20] H. R. MiLLER, Kervaire invariant one, Sém. Bourbaki (63ème année), 2010-2011, no. 1029.

[21] J. W. MiLnOR, On manifolds homeomorphic to the 7-sphere, Annals of Math. 64 (1956), 399-405.

[22] _ The Steenrod algebra and its dual, Annals of Math. 67 (1958), 150-171.

[23] , Differentiable structures on spheres, Amer. J. Math. 81 (1959), 962-972.

[24] J. W. Morgan, The Poincaré Conjecture, International Congress of Math. Zürich, Eur. Math. Soc., vol. I, 2007, pp. 713-736.

[25] Reviel Netz and William Noel, The Archimedes Codex, Orion Books Ltd., Phoenix, 2008.

[26] H. Poincaré, Analysis Situs; J. de l' École Polytechnique 1 (1895), 1-121.

[27] L. S. PontRJAGIN, Characteristic cycles on differentiable manifolds, Math. Sbornik (N.S.) (63) 21 (1947), 233-284 (Amer. Math. Soc. Translations Series 1, 32).

[28] __ Smooth Manifolds and Their Applications in Homotopy Theory, Trudy Mat. Inst. im Steklov, 45 Izdat. Akad. Nauk. S.S.S.R., Moscow, 1955 (Amer. Math. Soc. Translations Series 2, 11, 1959).

[29] D. C. RAVENEL, The nonexistence of odd primary Arf invariant elements in stable homotopy theory, Math. Proc. Cambridge Philos. Soc. 83 (1978), 429443.

[30] , Complex Cobordism and Stable Homotopy Groups of Spheres, Pure and Appl. Math., 121, Academic Press, 1986; 2nd ed., AMS Chelsea, 2004.

[31] W. W. Rouse BALL, Mathematical Recreations and Essays, Macmillan, 1959.

[32] V. P. SNAITH, Review of Some Applications of Topological K-theory by N. Mahammed, R. Piccinini, and U. Suter, Bull. Amer. Math. Soc. 8 (1), (1983), 117-120.

[33] V. P. SNAith and J. TORnehave, On $\pi_{*}^{S}(B O)$ and the Arf invariant of framed manifolds, Proc. Oaxtepec Conference in Honour of José Adem, Contemporary Mathematics, Amer. Math. Soc., 12, 1982, pp. 299-314.

[34] V. P. SNAITH, Stable Homotopy around the ArfKervaire Invariant, Birkhäuser Progress on Math. Series 273, 2009.

[35] N. E. STEENRoD, Cohomology Operations, Annals Math. Studies, 50 (written and revised by D.B.A. Epstein), Princeton Univ. Press, 1962.

[36] R. Tном, Quelques propriétés globales des variétés différentiables, Comm. Math. Helv. 28 (1954), 17-86. 